\title{
DESIGN DA INFORMAÇÃO EM SISTEMA DIGITAL PARA MAPEAMENTO DOS SITIOS DE ENDOMETRIOSE
}

\author{
Cristina Portugal \\ PUC-Rio \\ crisportugal@gmail.com \\ Marcelo Pereira \\ PUC-Rio \\ marcelopereira@me.com \\ Mariana Corrêa \\ PUC-Rio \\ antigonecarter@yahoo.com.br \\ Ricardo Lasmar \\ UFF \\ lasmar@ginendo.com
}

\begin{abstract}
Resumo: O presente artigo apresenta um projeto de pesquisa de caráter interdisciplinar entre os campos do Design e da Medicina, o qual foi contemplado pelo Edital FAPERJ № 41/2013 ${ }^{1}$ - Programa Apoio a Grupos Emergentes de Pesquisa no Estado do Rio de Janeiro - 2013. O projeto visa desenvolver tecnologicamente um sistema interativo digital para auxiliar a equipe cirúrgica no ato operatório para tratamento de pacientes com endometriose. Ele nasce da experiência da equipe de Ginecologia do Hospital Universitário Antonio Pedro e do Departamento Materno-Infantil da Universidade Federal Fluminense, com a colaboração do Laboratório Interdisciplinar de Design/Educação do Departamento de Artes \& Design da PUC-Rio. Em 2013, o trabalho que deu origem a este projeto ganhou o prêmio de melhor pôster no congresso da ESGE (European Society of Gyneacology Endoscopy) realizado na Alemanha. Referendado em sua versão analógica, o objetivo deste projeto de pesquisa é elaborar um sistema interativo digital que possibilite a visualização de dados de maneira clara e efetiva através de recursos gráficos. Ou seja, criar em formato digital o modelo analógico existente, permitindo o mapeamento visual que corresponda, de forma prática e objetiva, às áreas de acometimento pela endometriose, que faça parte do registro clínico, e que possa ser usado no acompanhamento de pacientes portadoras da doença.
\end{abstract}

\footnotetext{
${ }^{1}$ Sistema digital para mapeamento dos sítios de endometriose. http://www.faperj.br/interna.phtml?obj_id=9813
} 
Palavras chave: Design da Informação, Visualização de dados, Interdisciplinar, Endometriose

\section{INTRODUÇÃO}

O presente artigo apresenta um projeto de pesquisa de caráter interdisciplinar entre os campos do Design e da Medicina, o qual foi contemplado pelo Edital FAPERJ № 41/2013 - Programa Apoio a Grupos Emergentes de Pesquisa no Estado do Rio de Janeiro - 2013, e que visa desenvolver tecnologicamente um sistema interativo digital para auxiliar a equipe cirúrgica no ato operatório para tratamento de pacientes com endometriose.

O projeto nasce da experiência da equipe de Ginecologia do Hospital Universitário Antônio Pedro e do Departamento Materno-Infantil da Universidade Federal Fluminense, com a colaboração do Laboratório Interdisciplinar de Design/Educação (LIDE) do Departamento de Artes \& Design da PUC-Rio. Em 2012, o professor Ricardo Lasmar (autor do diafragma que deu origem a este trabalho) e outros do Serviço de Ginecologia do Hospital Antonio Pedro publicaram, no International Journal of Gynecology and Obstetrics, o trabalho "Diagram to Map the Locations of Endometriosis". Em 2013, este trabalho ganhou o prêmio de melhor pôster no congresso da ESGE (European Society of Gyneacology Endoscopy) realizado em Berlim, Alemanha. Referendado em sua versão analógica, o objetivo do presente projeto de pesquisa é elaborar um sistema interativo digital desenvolvido para a WEB e que poderá ser executado em navegadores compatíveis com os padrões do W3C. O sistema poderá operar em modo on-line, com armazenamento das imagens em nuvem, ou em modo offline, com armazenamento local.

A parceria entre o campo do Design e o campo da Medicina, neste caso, tem por objetivo possibilitar a visualização de dados de modo a comunicar a informação de maneira clara e efetiva através de recursos gráficos. Ou seja, criar em formato digital o modelo analógico existente, permitindo o mapeamento visual que corresponda, de forma prática e objetiva, às áreas de acometimento pela endometriose, que faça parte do registro clínico, e que possa ser usado no acompanhamento de pacientes portadoras da doença.

Os aspectos metodológicos que servirão de guia a esta investigação, que tem um viés qualitativo, encontram-se apresentados no processo de pesquisa para o desenvolvimento do sistema digital para mapeamento dos sítios de endometriose:

\section{FUNDAMENTAÇÃO TEÓRICA, DESENVOLVIMENTO E JUSTIFICATIVA}

A endometriose segundo Lasmar (2012) é uma doença que acomete cerca de $10 \%$ das mulheres em idade reprodutiva, apresentando prevalência de 30 a $50 \%$ nas pacientes com infertilidade. Define-se pela presença de glândulas e estroma endometrial em sítios ectópicos. Encontra-se associada a quadros clínicos variáveis e evolução insidiosa e progressiva, interferindo na qualidade de vida e desempenho profissional destas mulheres.

Um dos maiores desafios na abordagem de mulheres com suspeita de endometriose é avaliar a extensão da doença e se há comprometimento funcional de 
órgãos pélvicos ou extrapélvicos. A partir da história clínica, exame físico detalhado e de exames complementares pode ser feito mapeamento do grau de acometimento da endometriose, visando buscar o melhor tratamento para cada caso, de acordo com os objetivos de cada paciente. Considerando-se que tenha sido realizada a propedêutica clínica adequada para rastrear a doença, os dados obtidos devem ser registrados de forma precisa e objetiva, pois vão servir de elemento fundamental na conduta terapêutica, particularmente no momento da abordagem cirúrgica mais complexa.

A informação é essencial no desempenho da atividade médica, bem como na coordenação das ações da equipe multidisciplinar e no planejamento e obtenção de recursos administrativos e financeiros necessários em cada situação. O registro clínico deve ser claro e preciso, de compreensão fácil entre os membros da equipe, de forma organizada e padronizada, atribuindo um significado no contexto em questão. A inclusão de um diagrama visual que represente os locais acometidos pela endometriose poderá servir de guia no momento da abordagem cirúrgica e no seguimento clínico posterior.

A necessidade em transferir todas as informações adquiridas no pré-operatório das pacientes com endometriose para o momento do ato operatório é extremamente importante, pois existe um longo intervalo de tempo entre o inicio da investigação e o ato operatório. Considerando que, em sua grande maioria de casos, o tempo operatório de pacientes com endometriose ultrapassa cinco horas de duração, sendo considerado um procedimento delicado e de alto custo, torna-se imprescindível que o diagnóstico da doença tenha sido claramente identificado para que o procedimento cirúrgico, que utiliza uma série de instrumentos e equipamentos específicos, ocorra dentro de um planejamento prévio para evitar intercorrências. O fato de a investigação ser realizada por equipe diferente da que realizará o procedimento cirúrgico, e ainda, o fato de normalmente a paciente percorrer diferentes instituições durante este processo, requer que seu histórico tenha sido devidamente registrado, pois a melhora da paciente está diretamente correlacionada à retirada de todos os focos de endometriose que foram identificados no pré-operatório.

Com a possibilidade da utilização de um sistema interativo digital apontando os sítios da endometriose, será possível, ainda no ambulatório - momento em que se dá a indicação cirúrgica -, o preenchimento deste mapa digital. Esse mesmo mapa será reproduzido posteriormente na sala de cirurgia, recapitulando as informações do préoperatório. Este recurso poderá auxiliar a equipe cirúrgica a ficar ciente da complexidade da cirurgia, mapear os focos da doença impedindo o esquecimento de algum sítio, assim como também, estabelecer o provável tempo operatório e riscos inerentes. Portanto, o "mapa digital" representa a memória da investigação do préoperatório funcionando como uma ferramenta importante no ato operatório.

Para a confecção do "mapa digital" (figura 1) foi necessário o envolvimento de profissionais de diferentes áreas, em um trabalho multidisciplinar, isto é, médicos ginecologistas, designers, programadores e profissionais da área de informática.

A partir do diagrama apresentado abaixo, será desenvolvido um produto para funcionar em ambiente digital facilitando sua divulgação e utilização tanto na área da saúde como no meio acadêmico. 


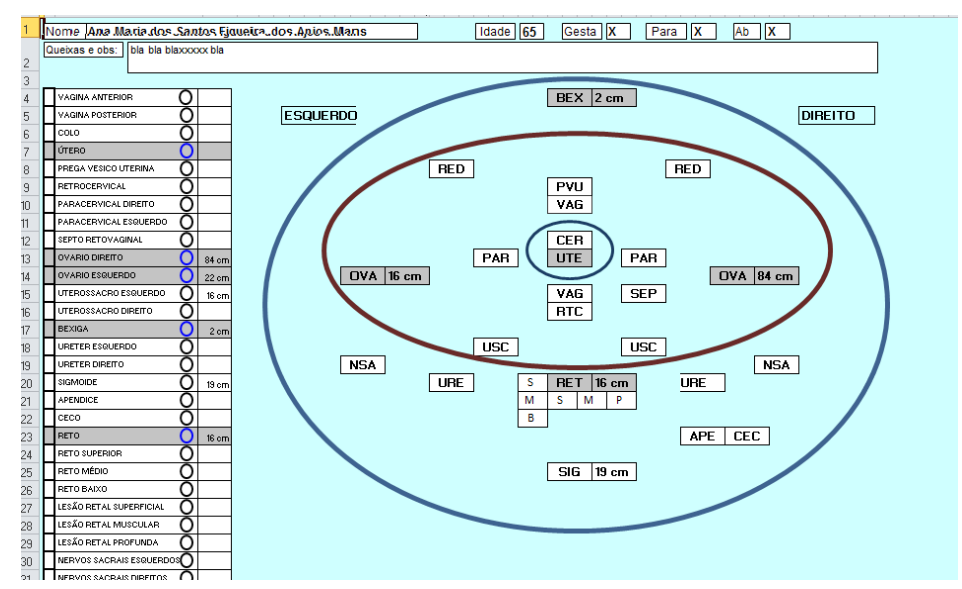

Figura 1 - Mapa analógico de Ricardo Lasmar et al. (2012)

No campo do Design, existe uma base teórica bastante abrangente das possibilidades de aplicação de seus métodos e técnicas para disponibilizar informação em ambiente digital. Para isso, vale aplicar os estudos que discutem questões sobre cor, tipografia, imagem, ícone, layout, dentre outros, que possibilitam a apresentação da informação mais significativa em interfaces digitais.

Segundo Portugal (2013), no Design de Interface evidenciam-se os elementos que permitem executar as operações, como por exemplo, a configuração e a organização de informações verbais, visuais, sonoras e sinestésicas, além dos índices de navegação e de interação. No entanto, a expressão visual da página segundo Dias et al. (2012), incluindo os elementos indicativos de interatividade, aparece como um conjunto de ícones, menus, linhas e outros elementos, que são dispositivos metafóricos de uma realidade operacional interativa acessível aos usuários.

Nesse sentido, ainda segundo os autores, essa visualidade, além de representar a realidade, também se constitui como um tipo de realidade. Os elementos visuais que aparecem no monitor compõem um espaço de ação, portanto cabe ao designer projetar os elementos de modo que induzam essas ações. Entretanto, para isso ser viável, torna-se necessário planejar de modo eficiente o processo simbólico de cada elemento, tendo em vista as características próprias, considerando a influência do contexto material e simbólico que circunda os elementos e usuários.

Em sistemas de hipermídia, os elementos de design para a orientação da navegação devem ter uma linguagem visual criada para atender os objetivos do projeto e as necessidades do usuário, dando força à mensagem de modo que seu reconhecimento seja imediato.

Navegar em ambientes que possuem diferentes linguagens para designar a mesma informação pode confundir o usuário durante a navegação fazendo com que ele perca o interesse no ambiente hipermidiático.

A utilização de hierarquias e repetições de elementos de design traz consistência e credibilidade para o usuário ao navegar em ambientes de hipermídia. Um bom sistema irá utilizar uma família tipográfica, o mesmo estilo em todos os ícones 
e uma hierarquia consistente de informações por meio de cores ou de outros elementos de design.

Os sistemas mais adequados de navegação são aqueles que vão diretamente ao ponto, sem excesso de informação. Os dispositivos devem disponibilizar apenas o necessário para a realização de tarefas.

Dentre as qualidades de uma navegação bem elaborada em um produto digital evidenciam-se três:

- rótulos claros: consiste na nomeação dos nós de navegação que devem ser claros e significativos para o usuário.

- consistência/inconsistência das ferramentas de navegação: está relacionada com a manutenção das características das ferramentas de navegação em todas as telas, em termos de aparência, localização e rotulagem, e a diferenciação entre ferramentas de navegação com funções diferentes.

- $\quad$ e clareza visual: refere-se ao uso adequado de elementos de design como cor, tipografia, imagem, layout etc. visando facilitar a navegação.

O "mapa analógico" já desenvolvido, que servirá de base para este projeto de pesquisa, está sendo testado também por oncologistas, buscando demostrar no préoperatório a extensão da doença e o provável estadiamento desta. Com o sistema digital, a sua utilização poderá ser ampliada para outras áreas médicas.

Com a publicação e premiação do trabalho no congresso internacional realizado na Alemanha, professores europeus realçaram a importância em desenvolver a alternativa em meio digital para que o mapa pudesse ser utilizado também em outros países. A finalização deste projeto desenvolvido por pesquisadores de universidades brasileiras permitirá que pesquisadores em geral, inclusive de outras nacionalidades, realizem trabalhos multicêntricos nacionais ou internacionais fomentando a pesquisa no país.

\section{METODOLOGIA DA PESQUISA}

Os aspectos metodológicos que servirão de guia a esta investigação, que tem um viés qualitativo, encontram-se apresentados no processo de pesquisa para o desenvolvimento do sistema digital para mapeamento dos sítios de endometriose:

- Pesquisar, à luz de metodologias de Design, meios para a construção de sistema digital para mapeamento dos sítios de endometriose.

- Como apontam Rosenfeld e Morville (2006), desenvolver a arquitetura de informação do ambiente digital segundo características, as quais são divididas em quatro grandes sistemas interdependentes, cada um composto por regras próprias e suas aplicações: 1. Sistema de Organização (Organization System): determina o agrupamento e a categorização do conteúdo informacional; 2. Sistema de Navegação (Navigation System): especifica as maneiras de navegar, de se mover pelo espaço informacional e hipermidiático; 3. Sistema de Rotulação (Labeling System): estabelece as formas de representação, de apresentação da informação, definindo signos para cada elemento informativo; 
4. Sistemas de Busca (Search System): determina as perguntas que o usuário pode fazer e o conjunto de respostas que irá obter.

- Desenvolver os componentes de navegação como menus, setas, nós de navegação, hipertextos, imagens. O sistema de navegação é, por sua vez, a forma de busca, relação e construção de conhecimentos do sistema digital e possui os seguintes objetivos fundamentais: Promover a orientação local e global do usuário dentro do próprio sistema; permitir o deslocamento do usuário entre os nós de navegação, de acordo com seu interesse e necessidade informacional; fornecer ferramentas de acesso à informação alternativa, à navegação nó a nó.

- Desenvolver mapa digital para plataforma Windows e IOS - (Desenvolvimento de projeto sob a perspectiva de metodologias do campo do Design).

- Entrevistar grupos selecionados de professores e residentes da UFF, e profissionais da área médica. A partir das observações feitas sobre o uso do mapa digital, os resultados serão analisados para reforçar, exemplificar e fundamentar a questão da eficácia no uso do produto. (Entrevistas semiestruturadas).

- Elaborar recurso digital a partir de um instrumento analógico para aplicação em ambulatórios e em cirurgias para tratamento de pacientes com endometriose. Com a participação de equipe multidisciplinar, profissionais das áreas de saúde, design e informática, será desenvolvido um instrumento, o qual será testado em procedimentos cirúrgicos no Hospital Universitário Antonio Pedro.

- Validar mapa digital junto a professores, médicos e alunos da UFF. (elaboração de roteiro de validação e referencial de análise).

- Durante a concretização do projeto e principalmente com sua conclusão, planeja-se a divulgação nacional e internacional do novo sistema interativo digital, buscando principalmente sua aplicação para grupos de assistência universitária e pública.

\section{PRIMEIROS RESULTADOS}

De acordo com Bonsiepe (1997), a interface deve ser entendida em um caráter mais amplo desde a sua ação instrumental, relacionada ao desenvolvimento de produtos, até sua ação comunicativa, uso de signos e informações. O autor ressalta que uma das características da hipermídia que a torna interessante é a interação dialógica do meio visual com o discursivo. Nela, a informação pode vir sob a forma de texto, áudio, imagens, sequências de vídeos, animações, música e sons (BONSIEPE, 1997, p. 144). Entretanto, estas formas precisam ser elaboradas dentro do processo de desenvolvimento de uma interface, pois se as possibilidades abertas pela tecnologia de informação e comunicação estarão ou não disponíveis, dependerá da qualidade de interface. Esta poderá inibir ou facilitar o acesso às informações, dependerá se a organização e apresentação de dados e a sua transformação em informações são válidas e significativas, e que possibilite a ação efetiva na comunicação.

Diante do exposto, o primeiro protótipo do aplicativo desenvolvido do sistema digital para mapeamento dos sítios de endometriose a luz do Design da Informação é 
apresentado a seguir. Estes layouts não são definitivos, mas já mostram um pouco das funcionalidades que estarão disponíveis na interface.

A primeira imagem (figura 2) é uma visão geral da interface. Os retângulos com as siglas são botões com três estados, um desativado, e dois ativados. Com um clique o usuário define um estado e, com um segundo clique, altera para o segundo estado. Cada estado é representado com uma cor diferente. Os campos em azul ao lado de alguns botões (BEX, OVA, RET e SIG) são para entrada de valores numéricos. No topo da página, temos campos para cadastro da paciente.

A barra cinza abaixo da caixa de texto serve para que o usuário possa expandir a ficha do paciente. Lá, haverá informações adicionais que ainda não foram definidas.

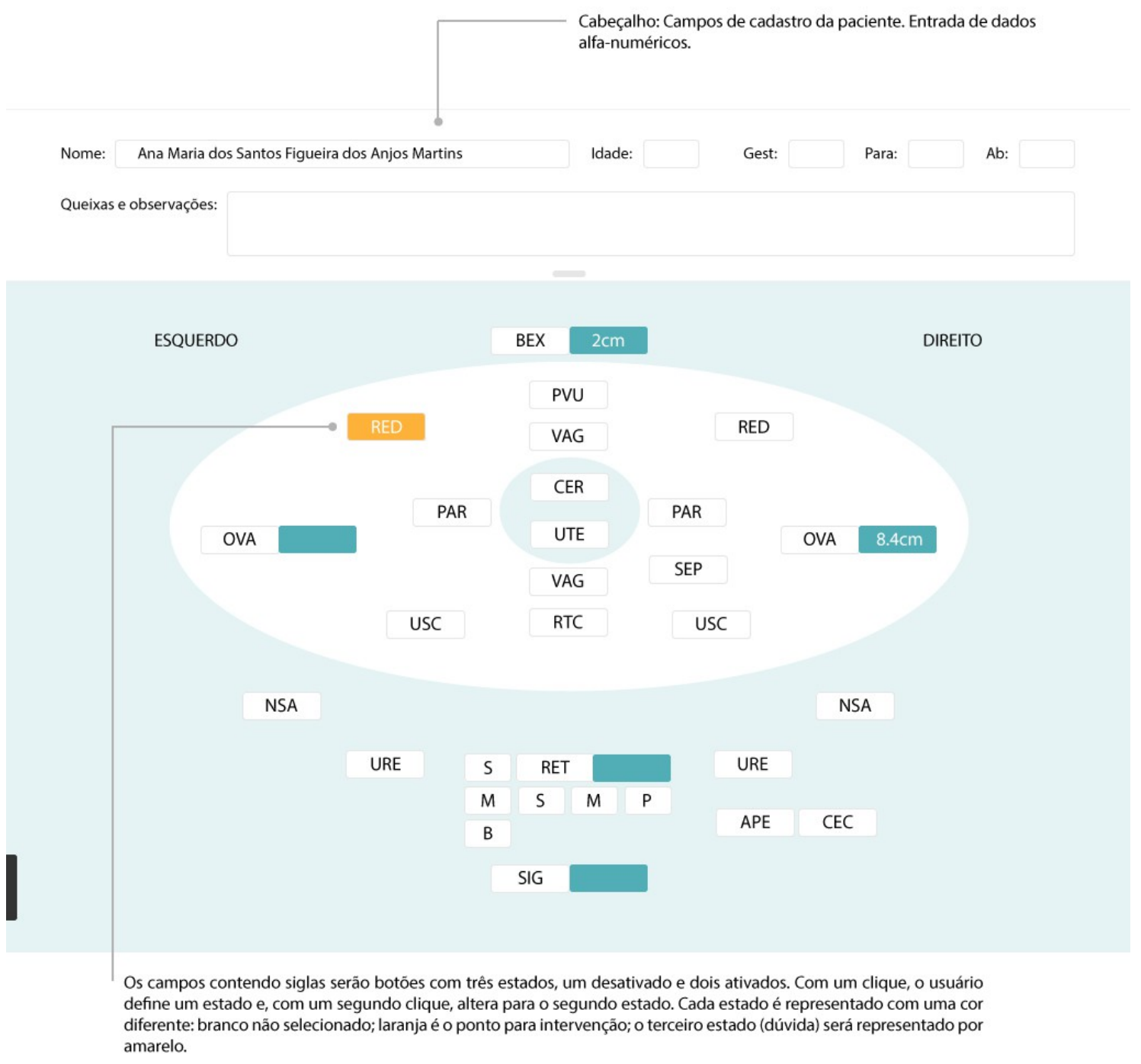

\section{Figura 2 - Interface do sistema interativo digital para mapeamento dos sítios de endometriose}

A entrada de valores numéricos pode ser realizada de duas formas: através da digitação convencional ou através de um slider circular (figura 3). Com um clique no campo, aciona-se o modo de entrada de dados (teclado). Ao clicar e arrastar sobre o campo, aciona-se o modo de entrada por slider. O menu lateral possibilita as seguintes 
opções: exibição em tela cheia, inserir imagem e travar a entrada de dados para que não sejam feitas alterações acidentais.

A entrada de valores numéricos pode ser realizada de duas formas: através da digitação convencional ou através de um slider circular (apenas para a versão Tablet). Com um clique no campo, aciona-se o modo de entrada de dados normal. Ao clicar e arrastar sobre o campo, aciona-se o modo de entrada por slider.

Nome: Ana Maria dos Santos Figueira dos Anjos Martins

Idade:

Gest:

Ab:

Queixas e observaçōes:

ESQUERDO

BEX

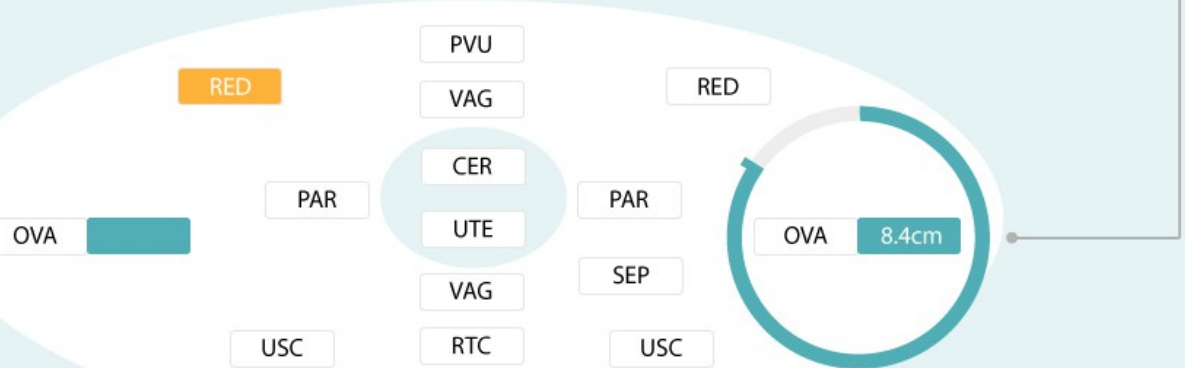

' $\mathbf{x}]$

NSA

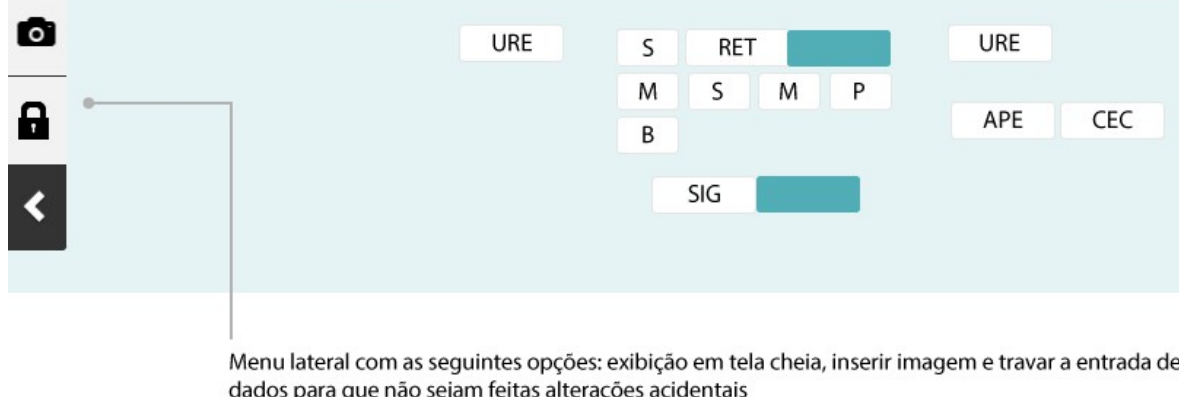

Figura 3 - Interface do sistema interativo digital para mapeamento dos sítios de endometriose

A inserção de imagem serve para que o médico possa colocar uma foto de fundo sob o mapa gráfico, facilitando a identificação das áreas com problemas. A imagem (figura 4) mostra um exemplo de uma foto aplicada. Como não temos como prever o tamanho da imagem que o médico irá inserir, é necessário permitir o redimensionamento da foto, quando necessário. 


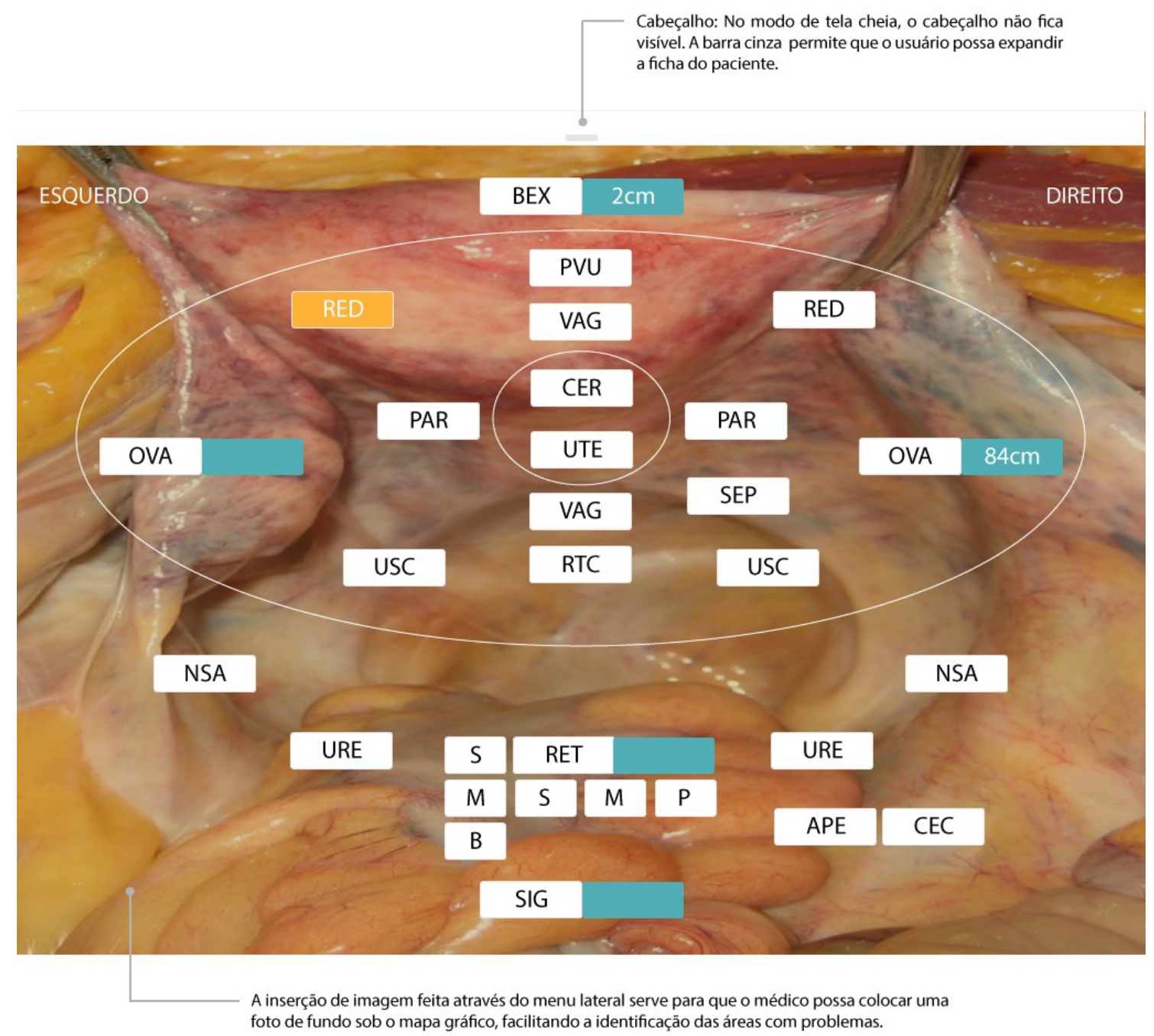

\section{Figura 4 - Interface do sistema interativo digital para mapeamento dos sítios de endometriose}

O aplicativo será desenvolvido para rodar em tablets, mas a primeira versão rodará apenas em desktop. Para a versão desktop iremos considerar a entrada de dados apenas como digitação de números, já que não é possível contar com um dispositivo touch. É por isso que a interface oferece recursos híbridos, compatíveis com ambos os formatos.

Com o protótipo desenvolvido, o sistema passará por teste de usabilidade, que é uma técnica formal que seleciona alguns usuários de modo a representar o grupo alvo para quem se destina o sistema. Estes usuários (professores, médicos e alunos da UFF) são designados a desenvolver tarefas no sistema em desenvolvimento de modo que se possa coletar dados a fim de, posteriormente, poder analisá-los e procederem às devidas correções ou alterações no sistema para que este atinja um melhor grau de usabilidade.

Como justificativa da importância dos testes de usabilidade junto aos usuários toma-se por base as ideias de Cybis, Betrol e Faust (2007), que afirmam que o objetivo 
de utilizar o recurso de testes de usabilidade e avaliação ergonômica é avaliar a qualidade das interações levando em conta os resultados dessas avaliações para a construção de novas versões das interfaces. Tal estratégia se faz necessária com a implementação desde o início do projeto, podendo reduzir o risco de falhas conceituais e garantindo que a cada ciclo o sistema responda cada vez melhor às expectativas e necessidades dos usuários em suas tarefas.

\section{CONSIDERAÇÕES FINAIS}

De acordo com Shedroff (2010), uma das habilidades mais importantes para quase todas as pessoas adquirirem na próxima década será aquela que permita criar informações significativas, interessantes e válidas. Para fazer isso, temos de aprender as maneiras de criar novas formas de organização e apresentação de dados e informações. $O$ autor afirma que faz pouca diferença se as nossas ferramentas de comunicação são os produtos tradicionais de impressão, produtos eletrônicos, programação televisiva, experiências interativas, performances ao vivo. Também não importa se estamos empregando dispositivos físicos ou eletrônicos ou nossos próprios corpos e vozes, pois o processo de criação é praticamente o mesmo em qualquer mídia. Essas questões se aplicam a todos os tipos de mídia e de experiências, porque elas tratam diretamente sobre o fenômeno da sobrecarga de informações, da ansiedade de informação, da literacia midiática, da imersão de mídia, e da sobrecarga tecnológica, enfim tudo o que necessita de melhores soluções.

Ainda segundo o autor, o cruzamento dessas questões pode ser resolvido pelo processo de Design da Informação e de Interação. Para Shedroff (2010), a compreensão do Design da Informação começa com a visão que a grande quantidade de coisas que bombardeiam nossos sentidos, todos os dias, não são pedaços de informação, mas simplesmente de dados.

Os dados se constituem em elementos tipicamente quantitativos e mensuráveis, podendo ser facilmente manipulados por processos computacionais quando codificados na linguagem digital. Dessa forma, podemos aplicar o poder computacional para criar formas de mapeamento desses dados, a fim de incorporar novos sentidos interpretativos.

A visualização de dados complexos em ambientes digitais, ou seja,
visualização dinâmica de dados é uma das formas culturais genuinamente
novas que se tornaram possíveis graças à computação. (...) Com os
computadores podemos visualizar conjuntos de dados muito mais amplos,
criar visualizações dinâmicas, alimentar dados em tempo real, basear as
representações gráficas de dados em sua análise matemática, usando vários
métodos, da estatística clássica à prospecção de dados, mapear um tipo de
representação em outro (imagens em sons, sons em espaços
tridimensionais, etc.) (MANOVICH, 2004, p. 149)

Como vimos, a propriedade essencialmente humana de estabelecer relações cognitivas entre conceitos de maneira visual já é explorada pelo Design da Informação. Tais estudos, por sua vez, ganharam ainda mais relevância quando suas pesquisas encontraram, naturalmente, aplicação na criação de interfaces para dispositivos computacionais, exigindo capacidade de exibição dinâmica e interativa. Dinâmica, no sentido de contemplar a capacidade de atualização dos dados ao longo do tempo e interativa, no sentido de oferecer ao indivíduo a possibilidade de escolher seus 
próprios caminhos de navegação e de maneira variáveis oferecidas pelo sistema. A dinamicidade e a interatividade são, portanto, duas características relevantes na análise de aplicações de visualização de dados e podem ser assim definidas: (FRY, 2002)

a) dinamicidade: ambientes dinâmicos pressupõem que alguma de suas propriedades internas varie ao longo do tempo, registrando alguma diferença perceptível quando o sistema é comparado em momentos distintos. A base de dados que compõe o sistema deve, portanto, estar preparada para registrar constantes interferências e evoluções;

b) interatividade: ambientes interativos pressupõe que um usuário pode aprender sobre um sistema de dados através da sua manipulação direta. A interatividade é um componente essencial da visualização de dados por confiar à ação do usuário a representação de estruturas complexas de dados. A capacidade de exibir ou esconder elementos, de aproximar ou afastar determinada área, de aplicar um filtro específico ou de refinar a pesquisa são recursos particularmente úteis para que um indivíduo obtenha um entendimento mais amplo do contexto oferecido pelos dados em questão.

Diante do exposto, este projeto de pesquisa de caráter interdisciplinar entre os campos do Design e da Medicina vem solucionar a questão da sobrecarga de informações por meio da visualização de dados de modo a comunicar a informação de maneira clara e objetiva. Isto não só possibilitará o registro clínico da paciente durante o ato operatório, como também poderá ser usado no acompanhamento de pacientes portadoras da doença.

O resultado esperado deste projeto é a criação de um sistema interativo digital bilíngue, inédito à luz de métodos e técnicas do Design da Informação, para ser utilizado em circuito nacional e internacional tanto para o registro do diagnóstico da endometriose quanto em cirurgias, quando o sistema ajudará a orientar na retirada de focos da doença, havendo a necessidade e a preocupação por parte dos especialistas de evitar ressecções incompletas e de avaliar cuidadosamente a possibilidade de multifocalidade e multicentralidade das lesões.

\section{AGRADECIMENTO}

A FAPERJ pelo auxílio financeiro.

\section{REFERÊNCIAS}

BONSIEPE, G. Design, cultura e sociedade. São Paulo: Edgar Blucher, 2011.

BONSIEPE, G. Design do material ao digital. Florianópolis: FIESC/IEL, 1997.

CYBIS, W.; BETROL, A. H. e FAUST, R. Ergonomia e Usabilidade - Conhecimentos, Métodos e Aplicações. São Paulo: Novatec, 2007.

DIAS, A. R. et al. Identidade de marca e simbologia na interface digital . Estudos em Design | Revista (online). Rio de Janeiro: v. 20 | no. 2 [2012], p. 1- 17.

FRY, B. Organic Information Design. Master of Science in Media Arts and Sciences, Massachusetts Institute of Technology. 2002. Disponível em < http://projects.ischool.washington.edu/tabrooks/424/OrganiclnformationDesign/thes0522d.pdf > Acesso em 10 mar. 2013. 
HASSENZAHL, M. User Experience (UX): Towards an experiential perspective on product quality. 2008. Não paginado. Disponível em: <http://www.marchassenzahl.de/pdfs/hassenzahl-ihm08.pdf>. Acesso em: 3 jun. 2012.

LASMAR R. B, et al, Diagram to map the locations of endometriosis, Int J Gynecol Obstet. 2012 Jul;118(1):42-6.

MANOVICH, L. Visualização de dados como uma nova abstração e anti-sublime. In:

LEÃO, L. (org.). Derivas: cartografias do ciberespaço. São Paulo: Annablume, 2004.

MOURA, M. Hypermedia Design: relationship between culture, language, transdisciplinarity and information. Selected Readings of the Information Design Internacional Conference, Recife, v. 1, p. 144-154, 2004.

PEREIRA, M. F. A ubiquidade computacional como ferramenta de ensino projetual em Design. Rio de Janeiro, 2012. 192p. Tese de doutorado - Departamento de Artes \& Design, Pontifícia Universidade Católica do Rio de Janeiro. 2012.

PORTUGAL, C. Design, User-Experience and Teaching-Learning. In: A. MARCUS. (Org.). DUXU 2014. 1ed. Suíça: Springer International Publishing, 2014, v. 3, p. 230-241.

PORTUGAL, C. Design, Educação e Tecnologia. Rio de Janeiro: Rio Books, 2013.

SHEDROFF, N. Information interaction Design: a unified field theory of Design. Disponível em: <http://www.nathan.com/thoughts/unified/>. Acesso em: 15 ago. 2012.

ROSENFELD, L.; MORVILLE, P. Information Architecture for the Word Wide Web. 3. ed. Sebastopol, CA: O'Reilly, 2006.

ULBRICHT, V. R. et al. Design de hipermídia: proposta metodológica. In: 30 Conahpa, 2008, São Paulo/SP. 3o Congresso Nacional de Ambientes Hipermídia para Aprendizagem, 2008.

WURMAN, R. S. Ansiedade de informação 2. São Paulo: Cultura, 2005. 